\title{
Experimental Validation of Particle-Based Discrete Element Methods
}

\author{
Catherine O'Sullivan ${ }^{1}$, Jonathan D. Bray ${ }^{2}$, and Liang Cui ${ }^{3}$
}

${ }^{1}$ Department of Civil and Environmental Engineering, Imperial College London, UK

${ }^{2}$ Department of Civil and Environmental Engineering, University of California, Berkeley, USA

${ }^{3}$ Department of Civil Engineering, University College Dublin, Ireland

\begin{abstract}
As a consequence of its particulate nature, soil exhibits a highly complex response to applied loads and deformations. Traditionally, geotechnical engineers have coupled continuum numerical analysis tools (such as the finite element method) with complex constitutive models to analyze soil response. This approach does not explicitly consider the particle-scale interactions underlying the macro-scale response observed in the laboratory and field. With increasing computational speeds, particle-based discrete element methods are becoming popular amongst geotechnical engineers in both research and practice. On a practical level discrete element methods are particularly useful for studying finite deformation problems, while from a more theoretical perspective they can be used to create virtual laboratories where the micro-mechanics of soil response can be analyzed in detail. This paper describes a series of validation studies that were performed to confirm that, despite their inherent simplifications, discrete element methods can accurately capture the macro-scale response of granular materials. It is shown that, once validated, these methods can provide useful information to explain the complex response exhibited by granular materials in conventional laboratory tests.
\end{abstract}

\section{Introduction}

The particulate, discontinuous nature of soil determines its unique and complex response to applied loads and deformations. However geotechnical engineers typically use continuum based analytical approaches, such as the finite element method in analysis. In continuum approaches relatively complicated constitutive models are required to capture the features of soil response that emerge as a consequence of its discrete nature. While the successful application of finite element analysis in geotechnical engineering is well documented (e.g. Potts, 2003), there is also a growing interest in the application of discrete element modeling approaches to analyze problems in geomechanics. Discrete element methods offer some advantages over continuum approaches when engineers are investigating the detailed mechanisms of finite deformation problems including earthquake surface fault rupture, anchor pullout, and mining subsidence. Simulations of conventional laboratory tests using the discrete element method can provide useful insight into soil response at the particulate level. Parameters that are difficult to measure in conventional laboratory testing (e.g. contact force evolution, particle rotation) can easily be monitored in these simulations. In addition, carefully controlled sensitivity analyses can be carried out to better understand the sensitivity of the response of granular materials to specific parameters.

Cundall (2001) provides a basic and general overview outlining the potential uses of DEM in numerical modeling in geomechanics, and reference to recent conference proceedings, e.g. Garcia-Rojo et al (2005), gives an indication of the wide variety of ways that geotechnical engineers are using various discrete element methods to advance their understanding of soil response. The objective of this paper is to describe a series of validation studies that have been completed to explore the ability of discrete element models to replicate accurately the response observed in carefully designed laboratory tests. The paper firstly presents an introduction to discrete element modeling and discusses the results of some recent research that demonstrates the usefulness of discrete element modeling in soil mechanics research. Then the results of three different validation studies are presented. These studies demonstrate the accuracy with which a discrete element model can replicate a real physical system, while also illustrating the insight a discrete approach can provide into the particle-scale interactions underlying the observed macro-scale response. 


\section{Discrete Element Modeling in Geomechanics}

\section{An overview of discrete element modeling}

The basic idea in particulate based discrete element modeling, as originally proposed by Cundall and Strack (1979), is to model a granular material as an assembly of rigid particles. These particles are allowed to overlap at the points where they come into contact and a rheological model is inserted at this contact point to calculate the inter-particle contact forces. As illustrated in Figure 1 the rheological model is relatively simple, comprising orthogonal springs with sliders. The springs can be linear elastic, non-linear elastic or elasto-plastic. The slider in the contact normal direction is used to prevent tensile forces developing between the particles, while the slider in the tangential direction allows the particles to move relative to each other when the contact frictional strength is exceeded (calculated using Coulomb friction). Note that the model parameters can be set to allow some tension in the contact normal direction (analogous to cementation) and a cohesion term can also be specified in the tangential direction.

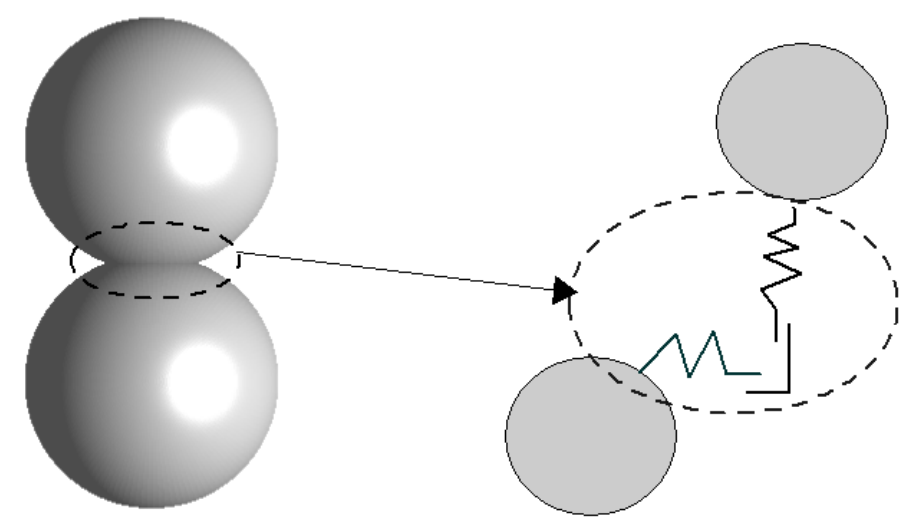

Figure 1. Modeling contact in discrete element simulations

A discrete element analysis is a transient, or dynamic, analysis. At each time step the total force acting on each particle is determined by considering both the contact forces and any body forces acting on the particle. These resultant forces are then used to calculate the incremental displacement for each particle for that time-step. As the contact forces are very sensitive to the calculated overlap, the geometry of the contact must be very accurately determined. Consequently the particles used in discrete element simulations are restricted to geometries that can be easily analytically described, usually disks in two dimensions and spheres in three dimensions. While more complex geometries, such as ellipsoids (e.g. Lin and $\mathrm{Ng}$ (1997), $\mathrm{Ng}$ (2004)) can be used, their use increases the computational cost of the simulations as a non-linear equation needs to be solved at each contact point at each time increment to determine the inter-particle forces.

In a discrete element method either implicit or explicit numerical integration approaches can be used. An example of an implicit discrete element approach is the method of discontinuous deformation analysis (DDA) (Shi, 1985). DDA was applied to particulate systems by Ke and Bray (1995). The analyses in this paper were all carried out using the distinct element method (DEM) algorithm, as proposed by Cundall and Strack (1979). A discussion on both implicit and explicit discrete element approaches is provided by O'Sullivan (2002). Users should be aware that an explicit DEM analysis is conditionally stable, consequently small time increments must be used in the analysis. O'Sullivan and Bray (2003) includes a discussion on the issues associated with selecting a suitable time-step for stable DEM analysis.

It should be appreciated that soil is a granular material and granular materials are of interest across a wide range of disciplines including physics, chemical engineering, geology and geotechnical engineering. Consequently discrete element modeling is used in a wide variety of applications. Reference to the recent proceedings edited by GarciaRojo et al (2005) presents the range of problems that discrete element methods can be used to analyze.

\section{Limitations of discrete element modeling}

Whereas discrete element methods are powerful tools for numerical analysis in geomechanics, it is important to appreciate their limitations. It can be argued that when granular materials are considered to be packed assemblies of 
particles, the mechanical interaction between particles is simple, and the material constants have explicit meanings, in contrast with the complexity associated with continuum modeling. However, the process of modeling grain geometry and detecting contact between particles is highly complex. In addition, development of accurate contact constitutive models for use in discrete element analysis is non-trivial, e.g. Thornton and Lanier (1997). The effective stress in a soil will govern its response and including pore water pressures in a discrete element framework is nontrivial.

A central limitation of discrete element modeling is the difficulty associated with modeling the large number of particles that exist within soil volumes large enough to be of practical interest to engineers. For example, consider a cylindrical specimen $38 \mathrm{~mm}$ in diameter and $75 \mathrm{~mm}$ high to be tested in a triaxial apparatus. If this specimen were filled with uniform $0.5 \mathrm{~mm}$ spherical particles at a void ratio of 0.585 it would contain almost 0.5 million particles. A real soil specimen, with a distribution of particle sizes would contain many more particles than this.

Recent advances in micro X-ray tomography allow us to visualize the internal three-dimensional structure of real soil (e.g. Oda et al., 2004) and particle image velocimetry technology (e.g. White et al., 2003) can give twodimensional information about the movement of soil particles during shearing. It is important to realize however that these methods cannot provide us with the detailed information about the distribution and evolution of the contact forces and internal stresses available in a DEM analysis. It can reasonably be anticipated that in the future DEM analyses will be coupled with micro X-ray tomography to give accurate information about the distribution of forces within a soil mass (e.g. Wang et al., 2004). The focus of the research presented here has been to quantitatively validate DEM models of granular material so that geotechnical engineers can be confident in using such models.

\section{Recent DEM contributions to Soil Mechanics}

Given the computational effort associated with DEM simulations, along with the inherent simplifications in a DEM model, it is useful to mention some of the ways in which DEM has been used to advance our understanding of the physics of soil response. Two representative examples illustrating the way DEM has advanced our understanding of soil response are the work of Thornton (2000) and Cheng et al (2003, 2004). In his study, Thornton studied the response of 3630 spheres subjected to a variety of loading conditions with periodic boundary conditions. Considering the simulations of axisymmetric compression tests, Thornton considered both loose $(e=0.660)$ and dense $(e=0.618)$ specimens of spheres and found that their response was qualitatively similar to the response of loose and dense sand. Thornton then performed an analysis of the particle scale interactions during the simulation. One aspect he examined was the contribution of the normal contact forces and the tangential contact forces to the overall deviatoric stress. As illustrated in Figure 2(a), the normal contact forces are the dominant contributors to the measured deviatoric stress. Thornton then examined the sensitivity of the material response to changes in the interparticle friction. These results, illustrated in Figure 2(b), illustrate that the material response is sensitive to the coefficient of interparticle friction, with the sensitivity decreasing at higher friction values. Thornton also examined the evolution of stresses and strains during non-radial deviatoric loading in detail and both Thornton (2000) and $\mathrm{Ng}$ (2004) have used DEM to examine the failure criteria used in soil mechanics.

Another interesting study is the work of Cheng et al $(2003,2004)$. In this research, crushable clusters of DEM spheres were developed by allowing tensile and cohesive forces to develop between the spheres (Figure 3(a)). The normal and shear stiffness parameters were assigned to the spheres by calibrating the crushing tests on the clusters against physical crushing tests. Their results indicate that for sands under isotropic compression, the response along the virgin compression line is dominated by particle crushing. Furthermore, the crushing of sand particles commences at overall stresses that are much lower than the crushing strength of the individual particles (Figure 3(b)). 


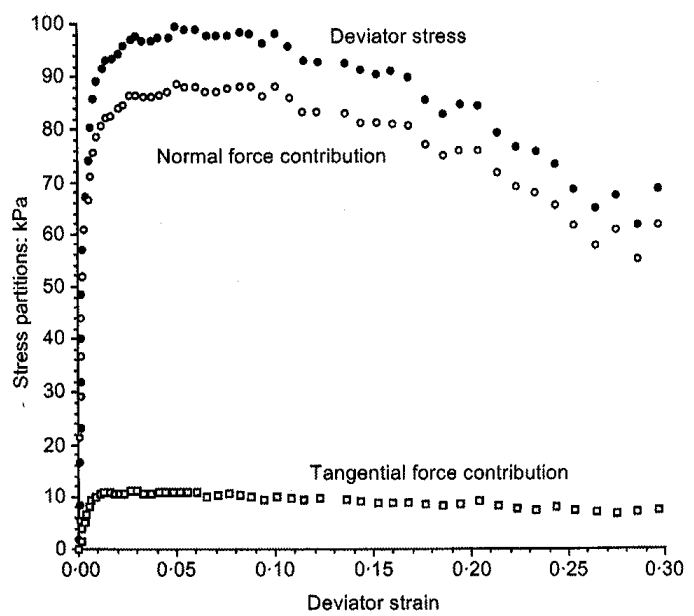

(a)

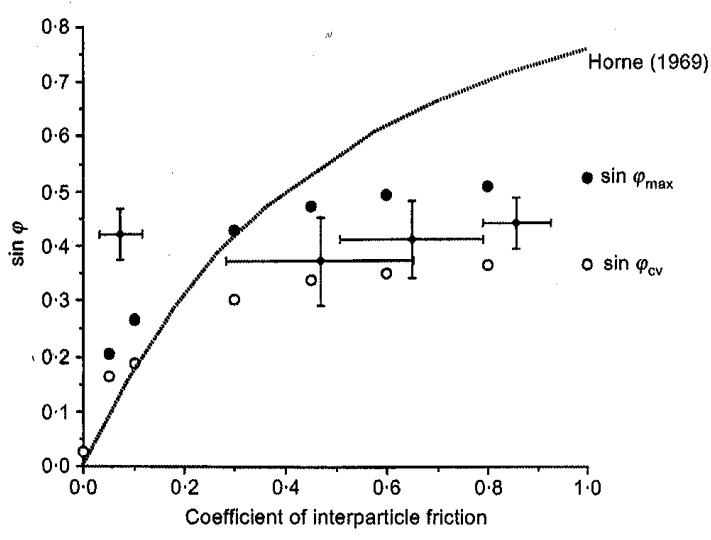

(b)

Figure 2. Contribution of Thornton (2000): (a) Evolution of normal and tangential force contributions to the deviator stress during axisymmetric compression (b) Effect of interparticle friction on mobilized angle of internal friction (Including theoretical results of Horne (1969) and experimental findings of Skinner (1969).

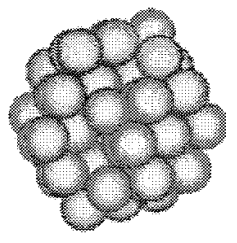

(a)

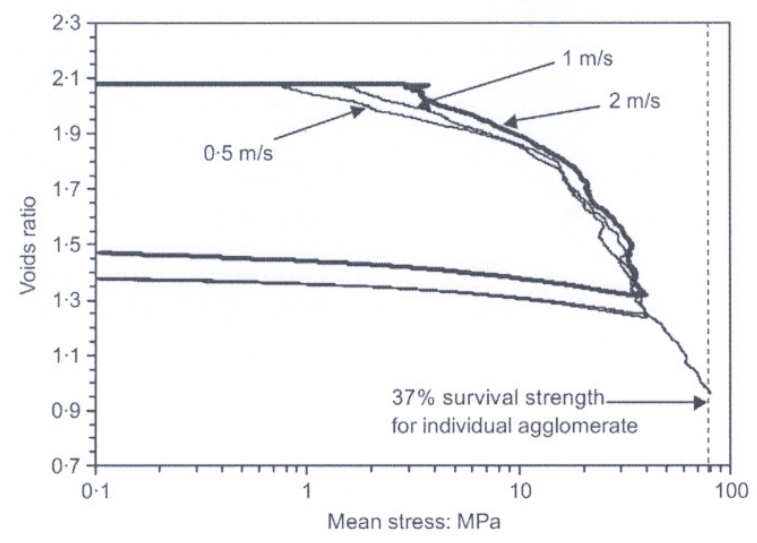

(b)

Figure 3. Contribution of Cheng et al (2003): (a) Illustration of typical crushable agglomerate (b) Isotropic compression and swelling.

Validation and Calibration of Discrete Element Codes

As proposed by Cundall (2000), amongst others, one approach to using DEM in geotechnical engineering is to calibrate DEM results (using idealized particle geometries) against the results of laboratory tests on real soils. In this calibration approach, the rheological model parameters are varied until the macro-scale response observed in the DEM model matches the field response. Examples of such an approach to calibration include Barla and Barla (2005) and Dolezalova et al (2002). The use of DEM in this manner should be approached with caution. It is the opinion of the authors of the current paper that it is not appropriate to vary the inter-particle coefficient of friction between the DEM particles to compensate for the differences in geometry between a real soil particle and a sphere. 
Friction inhibits sliding. The non-spherical, non-convex nature of real soil particles will result in multiple contact points between two contacting soil particles. Consequently, there will be effective transmission of moment between the particles, and rotations will be inhibited. Whereas the macro-scale responses may be similar, the particle-scale interactions of non-spherical particles will differ from the particle-scale interactions between highly frictional, spherical particles. The authors also query the use of two-dimensional particles to represent real soil grains. A real soil will develop contacts in the out of plane direction, as demonstrated below consequently the micro-mechanics will be different. The authors recognize the merit of carrying out two-dimensional DEM simulations where mechanisms can be more easily visualized, however assemblies of two-dimensional DEM particles must be considered as analogue soils.

This paper provides an overview of a series of four DEM validation studies. The overall aim of these studies has been to quantitatively demonstrate that a discrete element model can accurately capture the response of a real physical assembly of particles using the simple contact models discussed above (Figure 1). The specific objective of the first study was to provide a database of carefully controlled two-dimensional tests for validation of two-dimensional discrete element methods by considering disks with a regular hexagonal packing. The first threedimensional study directly extended the two-dimensional work by considering the response of regular assemblies of spheres. Having explored these regular assemblies we then progressed to a study of the micro-mechanics of the material response of randomly packed spheres in the direct shear test. By carefully comparing the macro-scale response observed in the simulations against laboratory test data, the conclusions regarding the particle-scale interactions in the direct shear test could be made with confidence.

\section{Two-dimensional Regular Disks in Biaxial Compression}

The first study considered was a two-dimensional study that examined the response of uniform and almost uniform rods in a hexagonal packing configuration. Hexagonally packed disks make up an idealized granular material that differs considerably from real sand. Two considerations motivated this study of disks in this lattice packing. Firstly, the specimen geometry could be captured accurately in the numerical simulation, so that a one-toone mapping was achieved between the particles in the physical specimen and the particles in the numerical simulation. In addition, when these regular packing configurations are considered, analytical expressions for the peak specimen strength are available. Therefore, a three-way comparison of theory, numerical simulations, and physical test results can be made, minimizing the potential for erroneous code validation (O'Sullivan and Bray, 2003).

A hexagonal packing arrangement is the densest packing configuration that can be achieved in two dimensions, and has frequently been considered in two dimensional granular mechanics studies (e.g. Rowe, 1962). The theoretical peak angle of mobilized friction $\left(\phi_{\mathrm{p}, \text { theory }}\right)$ of these assemblies can be estimated theoretically (e.g. Rowe, 1962, Thornton, 1979) and is given in Table 1. For these two dimensional systems, the angle of mobilized friction $\left(\phi_{\mathrm{m}}\right)$ is calculated as

$$
\phi_{\mathrm{m}}=\sin ^{-1}\left[\frac{\sigma_{1}-\sigma_{3}}{\sigma_{1}+\sigma_{3}}\right]
$$

where $\sigma_{1}$ is the total vertical stress and $\sigma_{3}$ is the confining pressure.

\section{Overview of Tests and Modeling Approach}

Two sets of laboratory tests and complementary numerical simulations were completed in this study and both are described in detail by O'Sullivan et al (2003). The first series of tests used borosilicate rods that were neither identical in size nor perfectly round, while the second series of tests used custom-ground steel rods. The current discussion will consider the tests on the steel rods only. The geometry of these steel rods was controlled to within a tolerance of $1 / 400 \mathrm{~mm}$. A number of different sizes of these steel rods were obtained to investigate the sensitivity of the response to minor variations in the rod size distribution. Nine discrete rod sizes were used ranging from $5.3 \mathrm{~mm}$ to $6.3 \mathrm{~mm}$ so that the sensitivity of the response of the system to minor perturbations to the system geometry could be assessed. 
Table 1. Characteristics of uniform disks and spheres with regular packing configurations (Rowe, 1962 and Thornton, 1979)

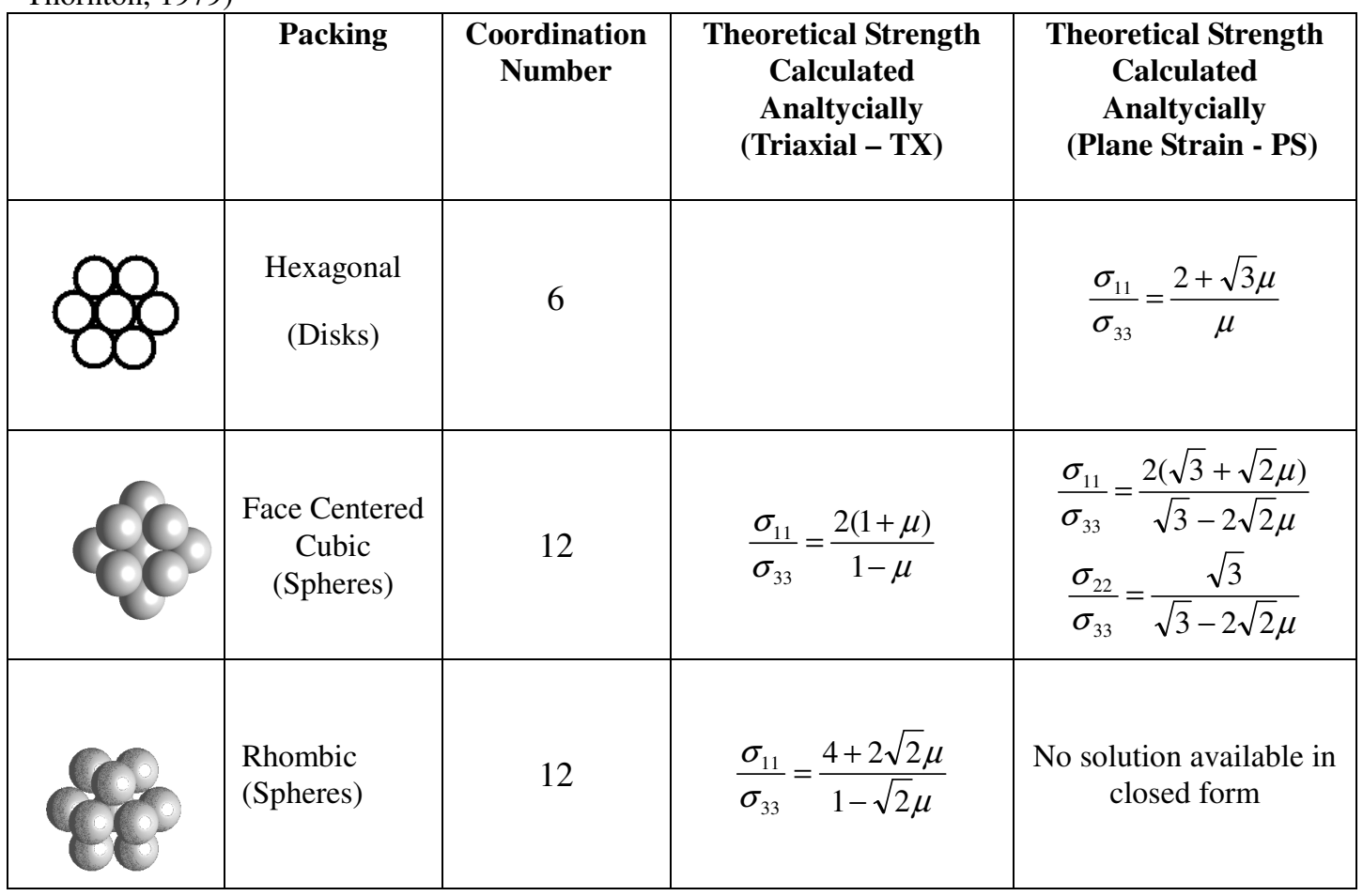

The coefficient of friction at the interface between the rod contacts is an important input parameter for the numerical analyses (refer to Figure 2). It is not possible to simply decide upon a friction coefficient based on knowledge of the material type. The coefficient of friction is influenced by the material type, the surface roughness, and the surface hardness. A series of sliding block type tests were performed on the steel rods used in this study and they yielded an average friction angle of $12.1^{\circ}$ and a standard deviation of $3.0^{\circ}$. Recognizing the variability in the results, the numerical analyses examined the sensitivity of the specimen response to variations in the surface friction characteristics.

The biaxial compression test procedure is described in detail by O'Sullivan (2002). The specimen of rods was confined using a flexible, latex membrane. A confining pressure was applied using a vacuum and the deviatoric load, LVDT reading of axial displacement, and confining pressure were recorded during the tests. For the nonuniform rod specimens the required distribution of rod sizes for the standard deviation of interest was determined using a random number generator and assuming a normal distribution.

The numerical simulations of the tests were performed using the two-dimensional discrete element code PFC-2D developed by Itasca (1998). The boundary conditions were implemented using the stress-controlled membrane approach described by Thomas and Bray (1999). The stress-controlled membrane applies forces to the particles on the outside of the specimens as if a pressure is being applied through a membrane. The top and bottom platens were modeled using rigid boundaries; the upper boundary was specified to move downward with a constant velocity to simulate the constant deformation rate applied in the laboratory. The deviatoric stress was calculated as the average of the contact forces acting on these rigid boundaries.

Linear springs were used in both the contact normal and tangential directions, and the shear spring stiffness and normal spring stiffness were both equal to $5 \times 10^{7} \mathrm{Nm}$. A mass density scaling factor of $10^{4}$ was used to increase the maximum stable time step for explicit analysis, as is common practice (e.g. Thornton, 2000), so the steel rods were simulated with a density of $7.85 \times 10^{7} \mathrm{~kg} / \mathrm{m}^{3}$. Sensitivity analyses in which the density was systematically varied confirmed that these quasi-static simulations were insensitive to particle density. A damping coefficient of 0.7 was used to model the energy loss in the physical contacts (Itasca, 1998). In a sensitivity analysis it was confirmed that the response was insensitive to the damping coefficient value used. Gravitational effects were neglected, because the applied external loads were much greater than the forces arising due to the weight of the rods. 


\section{Comparison of Physical Test and Simulation Results}

As described by Rowe (1962) and Thomas (1997), the deformation characteristics of specimens with this dense hexagonal arrangement differ significantly from the response of typical granular materials such as sand. Yet, these well-defined tests provide useful insights regarding the load-deformation response of particulate media, which need to be considered in numerical simulations. The results of some representative physical tests are compared with the results of some numerical simulations in Figure 4(a). The macro-scale response for both the tests and the simulations for uniform rods and with rods with particle size standard deviations of about $1.11 \%$ and $2.75 \%$ are shown. All of the specimens exhibited a qualitatively similar response; initially there was a stiff response, the angle of mobilized friction rose rapidly, the specimens sustained this peak strength for a short period, and then the mobilized strength decreased to zero when the specimen lost contact with the load cell following the closure of the shear band. The general observed trend for these tests is that the mobilized peak angle of friction decreases as the standard deviation of the distribution of rod sizes increases. This trend is also apparent in Figure 4(b), which shows the peak friction angle versus standard deviation of rod radii for all the tests and simulations considered by O'Sullivan et al (2003). As discussed by O'Sullivan et al (2003), tests repeated using slightly different rod configurations with similar standard deviations yielded similar peak strength values. Considering the numerical simulations, multiple "specimens" were created with two specified nominal standard deviations of radii (1\% and $2.75 \%$ ), to confirm that any variation in response observed was a function of the distribution of the radii sizes and independent of the specific configuration used. The numerical simulations captured the variation in peak response well. Both the numerical simulations and the physical tests indicate that the effect of the size deviation decreases with increasing size distribution. In a quantitative comparison, the numerical simulations yielded $\phi_{\mathrm{p}}$ values within $2^{\circ}$ of the corresponding physical test, for all cases.

It is important to realize that the numerical simulations incorporated accurately the inter-particle friction values. The initial back-analysis considered only the uniform-sized steel rods and assumed uniform surface friction angle of $12.1^{\circ}$, corresponding to the mean of the measured surface friction values. This analysis gave a $\phi_{p}$ value of almost $41^{\circ}$, which is close to the theoretical peak friction angle of $43^{\circ}$. A second numerical simulation incorporated the measured variation in the surface friction (i.e. standard deviation of $3.0^{\circ}$ ), gave $\phi_{\mathrm{p}} \cong 37^{\circ}$, which is much closer to the results of the physical tests, (i.e. $35^{\circ}-38^{\circ}$ ).

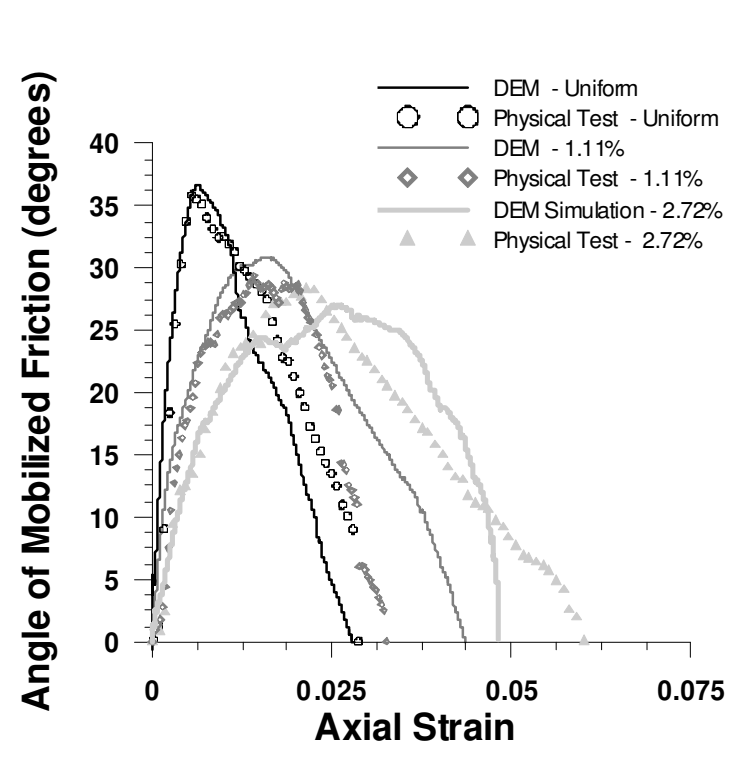

(a)

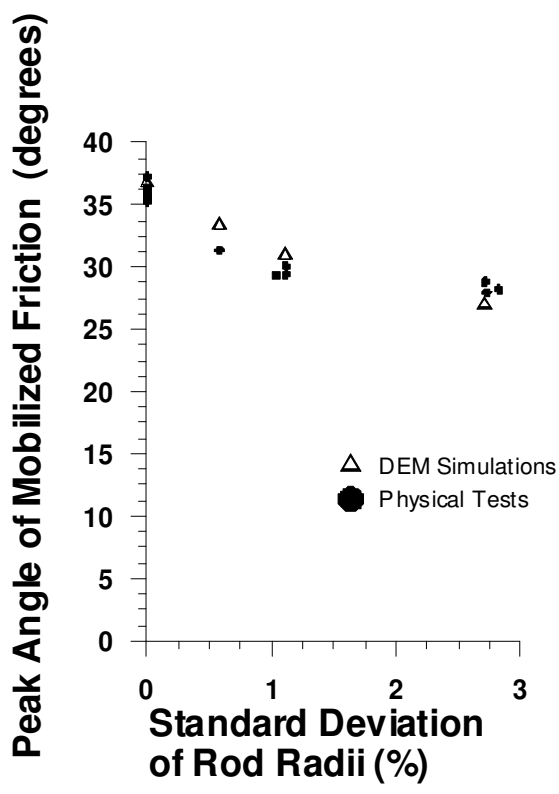

(b)

Figure 4. Response of disks with hexagonal packing configuration 
A series of simulations of the biaxial compression of $6 \mathrm{~mm}$ perfectly round rods (154 each), in which the standard deviation of the rod radii was systematically varied, was also performed. The trends in the results obtained were consistent with those of Figure 4. The peak strength of this assembly of rods is sensitive to minor variations in the distribution of disk radii. Increasing the standard deviation from $0.5 \%$ to $5 \%$ caused the calculated peak strength to decrease from $45^{\circ}$ to $25^{\circ}$. The decrease in strength can be explained by a decrease in the average coordination number, as illustrated in Figure 5(a). A hexagonal packing arrangement is the densest possible packing arrangement for disks. When the particles are not exactly the same size it is not possible to achieve this dense packing arrangement, so the number of contacts is reduced, and consequently there is a reduction in strength. The definition for coordination number adopted here is:

$$
Z=\frac{2 N_{c}}{N}
$$

where $\mathrm{N}_{\mathrm{c}}$ is the number of contacts and $\mathrm{N}$ is the number of particles. O'Sullivan et al (2003) interpreted the results using percolation theory. The variation in the initial distribution of the contact forces as a function of the different packings can be appreciated by reference to Figure 5(b), which illustrates the distribution of the contact forces for the uniform case and the case of a standard deviation of $2.7 \%$.

To explore the implications of this study's findings for two-dimensional validation studies using random, non-uniform particles, the effects of small variations in particle size on some simulations of biaxial compression of random arrangements of rods were examined. Numerical simulations were performed of one of the tests described by Thomas (1997), which comprised a mixture of 224 rods; $74(4 \mathrm{~mm})$ rods, $75(5 \mathrm{~mm})$ rods and $75(6 \mathrm{~mm})$ rods. The distribution of disk sizes about each nominal mean was systematically varied in a set of sensitivity analyses. As illustrated in Figure 6(a), the response of the assembly of rods was clearly very sensitive to small variations in the distribution of the radii sizes. A biaxial simulation of a large disk specimen, with 896 randomly arranged disks was performed to examine scale dependency. Three different disk sizes were used: $4 \mathrm{~mm}, 5 \mathrm{~mm}$ and $6 \mathrm{~mm}$. As before, the distribution of disk sizes about each nominal mean was systematically varied in a set of sensitivity analyses. As illustrated in Figure 6(b), for this larger assembly of rods the sensitivity to these minor geometrical differences is greatly diminished.

\section{Three-dimensional Regular Assemblies in Triaxial and Plane Strain Compression}

Recognizing that real granular materials are three-dimensional, these two-dimensional rod simulations were extended to three dimensions. In this a direct extension of the two-dimensional study, dense, regular assemblies of uniform spheres were considered; uniform spheres with face-centered-cubic (FCC) packing and with rhombic packing in compression tests. In three dimensions, for uniform-sized spheres, both these packings give the same maximum packing density and the same coordination number. As before these geometrical configurations were useful because the analytical expressions for the peak strength are known as given in Table 1. In addition the position of each sphere within the three-dimensional specimen was known, so the specimen geometry could be accurately replicated in the numerical simulation. In this direct extension of the two-dimensional study the response of, dense, regular assemblies of uniform spheres with face-centered-cubic (FCC) packing and rhombic packing in compression tests was considered. In three dimensions, both these packing configurations give the same maximum packing density and the same coordination number for uniform sized spheres. These tests were then simulated using a three dimensional DEM program. The study is described in detail by O'Sullivan et al (2004), and a representative subset of the data is presented here. 


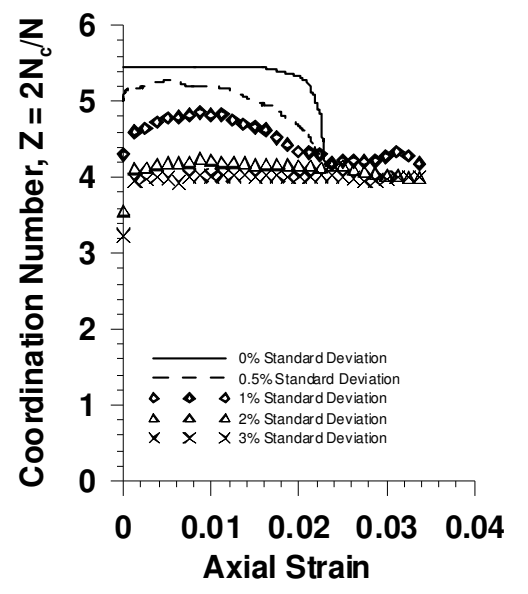

(a)

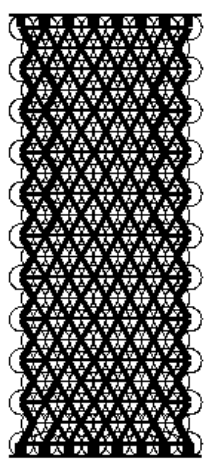

$0 \%$

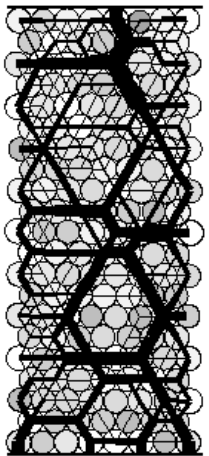

$2.7 \%$

(b)

Figure 5. Sensitivity of interparticle contact formation to small changes in geometry. (a) Variation in coordination number with axial strain for various size distributions (b) Initial distribution of contact forces for uniform rods ( $0 \%$ standard deviation) and rods with standard deviation of $2.7 \%$ (the line width is proportional to the magnitude of the contact force).

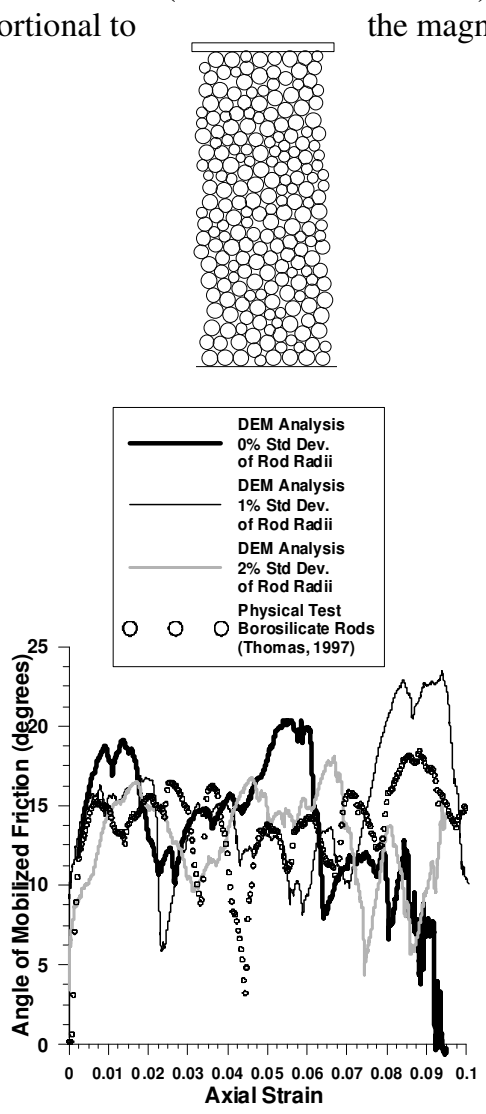

(a)
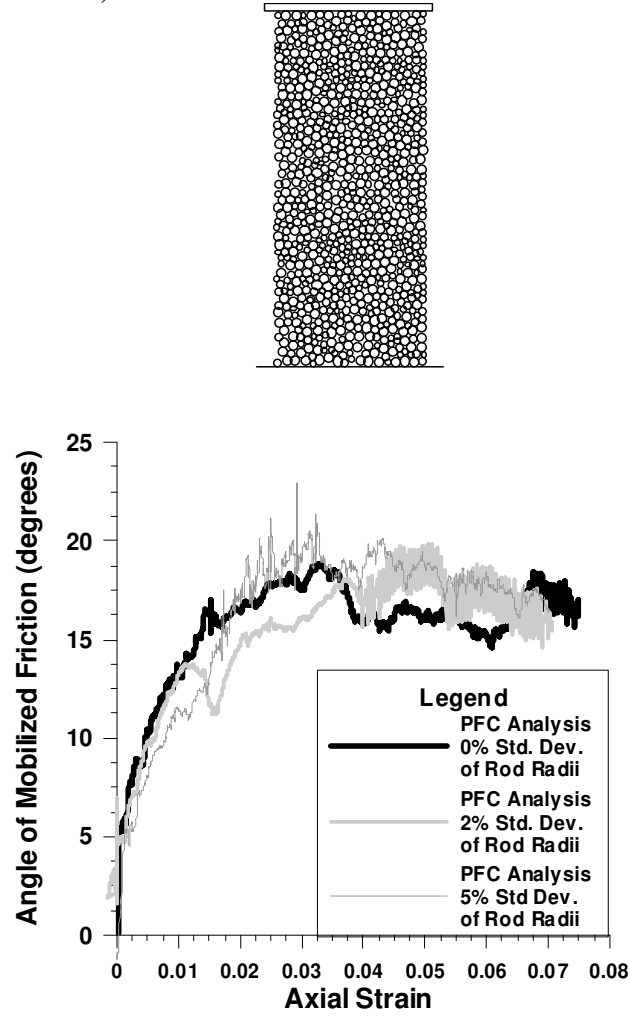

(b)

Figure 6. Sensitivity of random assemblies of disks to minor geometrical changes: (a) 224 disks (b) 896 disks. 


\section{Overview of Tests and Modeling Approach}

The physical test specimens were prepared by placing a latex membrane, sealed to a steel base cap, within a mold with a porous lining to which a vacuum was applied. The internal dimensions of the specimen mold were 12.07 $\mathrm{cm} \times 12.07 \mathrm{~cm} \times 33.0 \mathrm{~cm}$. The specimen cross-sectional areas were varied by inserting open-cell plastic sheeting between the mold and the membrane. For every specimen, the position of each ball was carefully monitored to ensure that the required packing configuration was achieved. The triaxial specimens were square in cross-section, while the plane strain specimens were rectangular in cross-section. Upon completion of the specimen construction, a top-cap was placed at the top of the specimen and the membrane was sealed against the top cap using a rubber oring. A vacuum was then applied to the specimen through the base, pulling the membrane in against the particles.

For the plane strain tests, two end plates were used to confine the steel ball specimens to plane strain deformation. The end plates were $25 \mathrm{~mm}$ (1 in.) thick plexi-glass. The interface between the test specimen and the plexi-glass plates was lubricated with silicone grease (Dow Corning high vacuum grease). The plates were connected using four instrumented, steel tie rods. The intermediate stress, $\sigma_{2}$, was calculated by dividing the sum of the tie-rod loads by the specimen area connecting the tie rods, and adding this component to the vacuum confinement. Following the findings presented previously, it was required that the ball geometry (i.e. the diameter and the sphericity) be tightly controlled. In addition, the ball surfaces were required to be uniform, and subject to a quantifiable tolerance in quality control. Grade 25 steel balls manufactured by Thompson Precision were selected. It was decided to use $12.700 \mathrm{~mm}$ diameter balls for the uniform tests, and to also obtain a sufficient number of balls with similar diameters so that the size distribution could be systematically varied. The manufacturing tolerance on both the ball diameter and sphericity was $\pm 0.0006 \mathrm{~mm}$.

As described by O'Sullivan et al (2003), accurate measurements of the interparticle coefficient of friction were performed using both the "four-ball" test, as described in ASTM D 4172-94, and a series of tilt tests. Based upon the findings of these tests our best estimate was that the particles had a mean friction angle of $5.50^{\circ}$, and standard deviation of $1.26^{\circ}$ and these values were used in the numerical simulations. Tilt tests were also carried out to measure the angle of friction between the steel balls and the boundaries. Considering the specimen base cap and top cap, the mean friction angle was found to be $6.52^{\circ}$, with a standard deviation of $1.14^{\circ}$. Along the lateral boundaries in the plane strain compression tests, the balls were enclosed by a latex membrane, which then contacted the lubricated plexi-glass plate. To measure the coefficient of friction for this interface, the friction specimen was placed upon the bottom cap, a piece of membrane was then laid over the specimen and sealed to the base cap using a rubber o-ring. A vacuum was then applied to the base cap, which pulled the membrane tight against the balls. The specimen was inverted and placed upon a lubricated plexi-glass plate for the tilt tests. Five specimens were prepared as described. For each specimen, the tilt test was repeated 5 times, giving a total of 25 tilt tests. The mean friction angle was found to be $1.92^{\circ}$, with a standard deviation of $0.67^{\circ}$.

The numerical simulations were performed using the 3D DEM code described by O'Sullivan (2002). This code is a modified version of Ellipse3D (Lin and $\mathrm{Ng}, 1997$ ), which in turn is a modified version of Trubal (Cundall and Strack, 1979). The initial stage of the analysis was to calibrate the numerical model. The objective of the calibration was to select the combination of analysis parameters that best matched the stress-strain response observed in just one type of physical test, i.e. the triaxial tests on the specimens with FCC packing. The parameters in question are the spring stiffness, $\mathrm{K}$, and the mass damping parameter, $\alpha$. For the simulations discussed here, the spring stiffness value was selected by attempting to match the initial "linear" section of the stress strain curve of the test data considering only one set of test data (i.e. the FCC triaxial case). Based on the results of this calibration study the DEM input parameters were as follows: a radius of $20 \mathrm{~L}, \mathrm{~K}=2.0 \times 10^{9} \mathrm{M} / \mathrm{T}^{2}, \rho=20000 \mathrm{M} / \mathrm{L}^{3}$, $\sigma_{\text {confining }}=50000 \mathrm{M} / \mathrm{LT}^{2}$, and $\alpha=0.3$.

The upper, lower, and lateral boundaries were modeled using planar, rigid boundaries. An automated "stress-controlled-membrane" was implemented in the DEM code for simulating the side membrane boundaries. The membrane calculates the force that should be applied to each ball by triangulating the boundary particle centroids to calculate the equivalent lateral force, which should be applied to the boundary balls. For all of the simulations described here, a measurement sphere, centered close to the specimen center, was created. The stress in this measurement sphere $\left(\bar{\sigma}_{i j}\right)$ was then calculated as:

$$
\bar{\sigma}_{i j}=\frac{1}{V} \sum_{c=1}^{N c} f_{i}^{c} l_{j}^{c} \quad(\mathrm{i}, \mathrm{j}=\mathrm{x}, \mathrm{y}, \mathrm{z})
$$

where $N_{c}$ is the number of contacts within the measurement sphere, $V$ is the volume of the sphere, $f_{i}^{c}$ is the contact 
force vector at contact $c, l_{j}^{c}=x_{j}^{b}-x_{j}^{a}$ is the branch vector connecting two contacting particles, $a$ and $b$ with centroids at $x_{j}^{a}$ and $x_{j}^{b}$. This measurement sphere was used in conjunction with a servo-controlled boundary algorithm to bring each specimen into an initial isotropic state.

\section{Comparison of Physical Test and Simulation Results}

Considering firstly the response of the uniform specimens in triaxial compression (as shown in Figure 7), the specimen response was similar to the response of the hexagonally packed rods described previously. Initially, the response was very stiff until the peak strength was mobilized. In the post-peak conditions, a distinct localization formed, which dilated as the deviatoric load decreased. For both specimen types, the localization was a single shear plane across the specimen. In contrast, for the rod tests, a shear band developed along two failure planes, separated by a single line of rods. This is most likely a result of the difference between the initial orientation of the plane along which initial sliding occurs and the specimen fabric. Also, in contrast to the rod tests, in which the post-peak drop in deviatoric stress was relatively rapid and monotonic, for the triaxial tests on balls the post-peak stress initially only decreased slightly prior to the occurrence of sudden failure. The initial rate of post-peak stress decrease for the rhombic specimens was slightly greater than that for the FCC specimens. Then, there was a sudden drop in stress at an axial strain value of about $1.25 \%$ for the rhombic specimens and about $1.8 \%$ for the FCC specimens. At this point, the specimen lost contact with the load cell.

The differences in strength as a function of packing are clearly seen here. Whereas Figure 7 includes the results of only two representative laboratory tests, a series of tests were performed to ensure the repeatability of the tests. Considering all the test data (given in O'Sullivan et al (2004)), the rhombic specimens yielded a mean peak angle of mobilized friction, $\left(\phi_{\mathrm{mob}}\right)$, of $41.6^{\circ}$, while the FCC specimens had a mean peak, $\phi_{\mathrm{mob}}$ of only $24.4^{\circ}$. For rhombic packing, in triaxial conditions, the theoretical peak $\phi_{\text {mob }}$ value is $41.6^{\circ}$, while for the FCC packing the theoretical peak $\left(\phi_{\mathrm{mob}}\right)$ value is $24.6^{\circ}$ (assuming a surface friction angle of $5.5^{\circ}$ ). The laboratory test results compared favorably with the theoretical estimates. The DEM simulations presented in Figure 7 explored the sensitivity of the response to the friction between the spheres and the boundaries. The first simulations assumed a smooth boundary with an interface friction angle of $0^{\circ}$, while the second simulations with "frictional" boundaries used the measured friction value of $6.5^{\circ}$.

Considering the differences between the laboratory test data and the numerical simulations, qualitatively, the shape of the post peak response for both simulations is similar to the physical test response for both packing geometries. Considering the FCC case, initially, after the peak, the stress drop was gradual for both simulations, slightly exceeding the rate of stress decrease observed in the physical tests. For the rhombic specimens, the numerical simulations matched the initial post peak rate of stress decrease closely. In the numerical simulations, as well as in the physical tests, after peak, the deviatoric stress decreased almost linearly up to a point where a sudden drop in stress occurred, and the specimen lost contact with the top cap. For the FCC specimens, both the numerical simulations underestimate the strain at which the specimen lost contact with the top platen, although using the frictional boundaries improves the comparison significantly. In contrast, the numerical simulations overestimated the strain at which this load "drop-off" occurred for the simulations of rhombic specimens. For both test types, while the DEM simulation can satisfactorily model the response observed in the laboratory up until the peak strength, the post-peak response is not captured as accurately (that is without readjusting the input parameters after the fact). It is important to emphasize that the numerical simulations first had to incorporate accurately measured particle surface friction (both mean and standard deviation) to achieve reasonable results. 


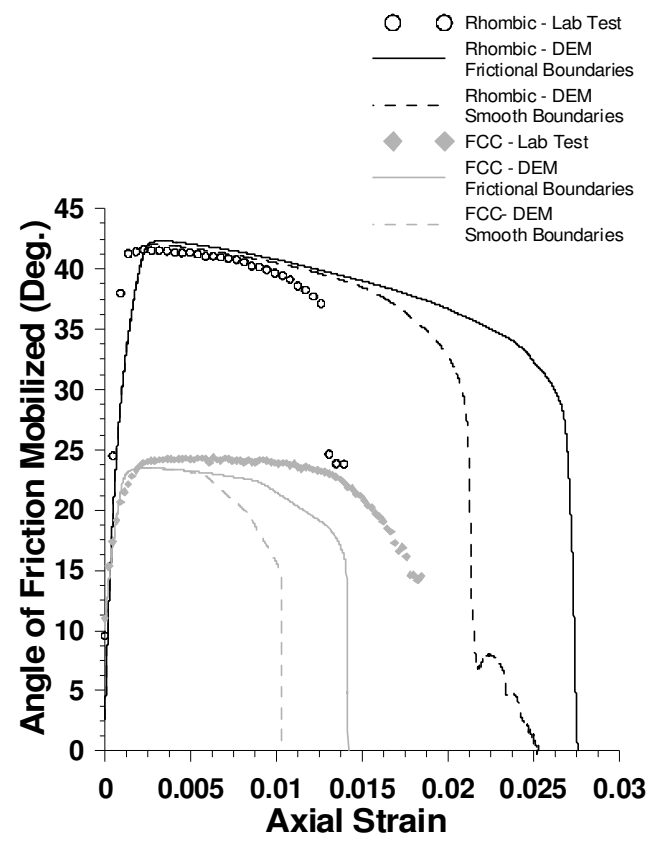

Figure 7. Response of uniform steel balls with rhombic and FCC packing configurations undergoing triaxial compression; comparison of laboratory test data and DEM simulations.

As with the two-dimensional study, the sensitivity of the response to perturbations in the specimen geometry was also explored. Considering the response of the spheres with rhombic packing in plane strain compression, the results for both the physical tests and numerical simulations are summarized in Figure 8. Considering firstly the physical tests, the particle size distribution was controlled by placing two thousand $12.700 \mathrm{~mm}$ diameter balls in a large container, and then introducing equal numbers of $11.906 \mathrm{~mm}$ and $13.494 \mathrm{~mm}$ grade 25 chrome steel balls to obtain mixes with different standard deviations of the particle size distributions. The non-uniform ball sizes were then distributed randomly through the regularly packed specimen. For the ball specimens, it was found that when the standard deviation exceeded $2 \%$, it was not possible to maintain a regular packing throughout the specimen height. Although there is some scatter in the data, the trend is consistent with that of the rod study; that is, the strength decreases with increasing standard deviation of the particle size distribution for both the triaxial and plane strain tests, as illustrated in Figure 8(a). The mean peak angles of mobilized friction were $40.0^{\circ}$ for the uniformsized ball tests, $37.5^{\circ}$ where the standard deviation of the ball diameters was $0.7 \%, 36.0^{\circ}$ where it was $1.2 \%$, and $32.6^{\circ}$ where it was $1.9 \%$. Whereas the major principal stress ratio decreased with increasing particle size distribution in the plane strain tests, the stress ratio $\sigma_{22} / \sigma_{33}$ increased, as illustrated in Figure $8(\mathrm{~b})$. There was also a definite variation in the shape of the response curve as a function of the standard deviation of the particle size distribution

\section{Three Dimensional Direct Shear Tests}

The studies presented above have considered very idealized test conditions and materials only. In an extension of this study we have also considered the response of randomly packed spheres in the direct shear test apparatus. The direct shear test is a finite deformation test, consequently it is well suited for DEM analysis. Earlier twodimensional DEM simulations of the direct shear test have clearly illustrated the potential to use DEM to develop an in-depth understanding of the particle-scale interactions in the direct shear apparatus (Masson and Martinez, 2001; and Zhang and Thornton, 2002). We also note that Dounias and Potts (1993) analysed the direct shear test using the finite element method. Some representative results from this study are presented here and further details are presented in Cui and O'Sullivan (2005). 


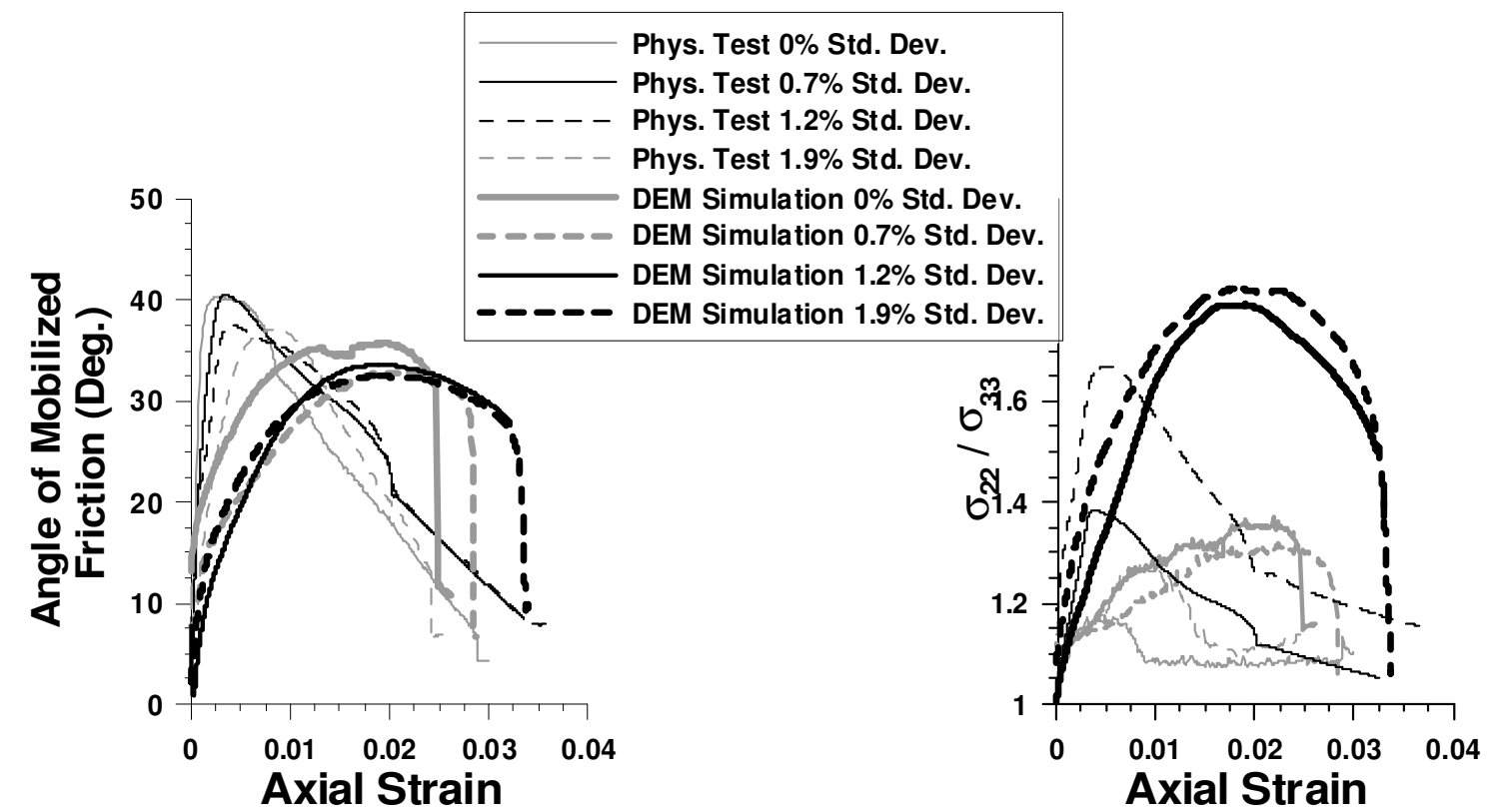

Figure 8. Response of uniform steel balls with rhombic and FCC packing configurations undergoing plane strain compression; comparison of laboratory test data and DEM simulations.

\section{Overview of Tests and Modeling Approach}

The physical tests used a standard direct shear test apparatus, comprising a metal box of square cross section (60 mm wide), divided horizontally into two halves. During testing, the lower section of the box was moved forward at a constant velocity $(0.015 \mathrm{~mm} / \mathrm{s})$ while the upper section of the box remained stationary. The force required to maintain the upper section of the box in a stationary position was measured using a load cell and proving ring. The normal (vertical) load was applied to the top of the shear box using a system of dead weights attached to a lever. An average shear stress value was calculated by dividing the horizontal load measured in the proving ring by the original specimen cross-sectional area and the normal stress was also calculated by dividing the applied normal load by the original specimen cross-sectional area. Throughout the discussion presented here the global shear strain $(\gamma)$ is considered to be the displacement of the lower section of the box divided by initial height of the box, while the vertical strain taken to be the ratio of the vertical displacement of the top of the shear box to the original height of the shear box.

As before, for this study Grade 25 Chrome Steel Spheres were used. In this study the sphere radii were 0.992 $\mathrm{mm}$. The average inter-sphere friction coefficient of 0.096 was assumed in the current study as the surface properties of the spheres were equivalent to the spheres considered in the physical tests presented previously. To replicate accurately the boundary conditions in the DEM model, the sphere-boundary friction coefficient for the apparatus used in the current study was measured in a series of 25 tilt tests, giving a value of 0.175 . A series of tests were performed, as discussed by Cui and O'Sullivan (2005), however in the current discussion only a single representative test performed under a normal stress of $164 \mathrm{kPa}$ is considered.

The 3D DEM program described above was used in the numerical simulations, however, in this case the elastic Hertz-Mindlin contact model was used to model the contact between spheres. The direct shear test simulation approach adopted here was originally proposed by O'Sullivan (2002) and is schematically illustrated in Figure 9. During the simulation, the specimen is enclosed by ten rigid boundaries, one horizontal boundary at the top, one horizontal boundary at the bottom, two vertical boundaries at the front, two vertical boundaries at the back, two vertical boundaries at the left, and two vertical boundaries at the right of the specimen. Prior to shearing, the two left boundaries the two right boundaries, the two back boundaries and the two front boundaries are each co-planar. During shearing, one set of vertical boundaries is moved laterally with a constant velocity. An imaginary horizontal plane is constructed through the middle of the specimen. All of the particles with centroids located above this plane are checked for contact with the moving boundaries, while all of the particles with centroids located below this plane 
are checked for contact with the stationary boundaries. Using this approach the computational effort associated with contact detection between a sphere and the corner of the shear box was avoided.

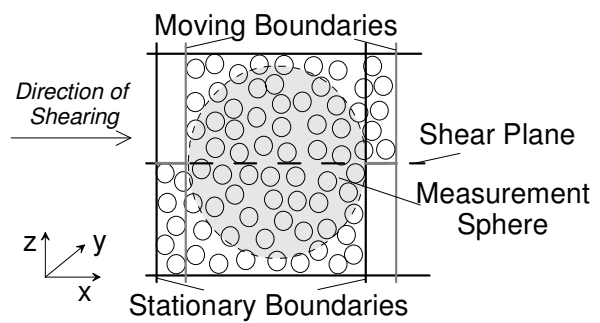

Figure 9. Schematic diagram of direct shear test simulation in DEM

\section{Comparison of Physical Test and Simulation Results}

Figure 10 compares the results of a representative laboratory test with a representative numerical simulation from this study. In the initial simulations it was difficult to obtain an exact match between the void ratios of the physical test specimens and the DEM specimens, with the DEM specimens tending to attain equilibrium (i.e. come to rest with a stable fabric) at lower void ratios in comparison with the physical test specimens. Consequently, a specimen generation procedure was developed, whereby a relatively loose assembly of particles was initially generated, then the particles were allowed to come to rest under a vertical body load, while varying the inter-particle coefficient of friction to achieve the desired void ratio. For the physical test presented here the specimen had a void ratio of 0.589 , while the void ratio in the numerical simulation was 0.532 .

Considering firstly the stress-strain response illustrated in Figure 10(a), relatively good agreement was attained between the DEM simulation and the physical test. The numerical simulation was slightly stiffer than the physical test and the peak stress was reached at a slightly lower shear strain in the numerical simulation, in comparison with the physical test. Considering the entire database of test results, the average angle of shearing resistance ( $\left.\phi^{\prime}\right)$ for the numerical simulations was found to be $23.8^{\circ}$ and the average $\phi$ ' for the physical tests was $24.1^{\circ}$. As illustrated in Figure 10(b), the numerical simulation also successfully captured the volumetric strain response of the specimen. The simulation exhibited slightly less initial compression and slightly less volumetric dilation in comparison with the physical test. The full study considered the sensitivity of the response to the method of specimen preparation, the interparticle friction values and the boundary friction, as discussed by Cui and O'Sullivan (2005).

\section{Micro-Scale Analysis}

As a good agreement was achieved between the numerical simulation results and the physical test data at a macroscale, the numerical simulations could be used to analyze the micro-scale interactions in the real physical system with some confidence. The full micro-scale analysis considered the fabric tensor, the orientation of the principal stresses, the rotations and the local strain distribution. In the current discussion a brief analysis of the inter-particle contact forces and the incremental particle displacements is considered.

To appreciate the spatial variation in the contact forces, two orthogonal views of the contact force network at a global shear strain $(\gamma)$ of $15.3 \%$ are illustrated in Figure 11. Figure 11(a) is a side view of the specimen and Figure 11 (b) is a front view of the specimen. In both cases, for ease of visualization, only the forces in the central fifth segment of the specimen are plotted. For additional clarity, only contacts where the magnitude of the contact force exceeds the average contact force plus one standard deviation are considered. The distribution of contact forces illustrated in Figure 11(a) is qualitatively similar to the distribution of contact forces obtained by both Masson and Martinez (2001) and Zhang and Thornton (2002) in their two-dimensional discrete element analysis of the direct shear test. The forces are transmitted diagonally across the specimen in the $\mathrm{x}-\mathrm{z}$ plane. An orthogonal view of the specimen, looking along the x-axis, is given in Figure 11(b). While it is difficult to identify any clear trend in the orientation of the contact force vectors from this perspective, the components of the contact forces orientated in the y-direction are finite. 


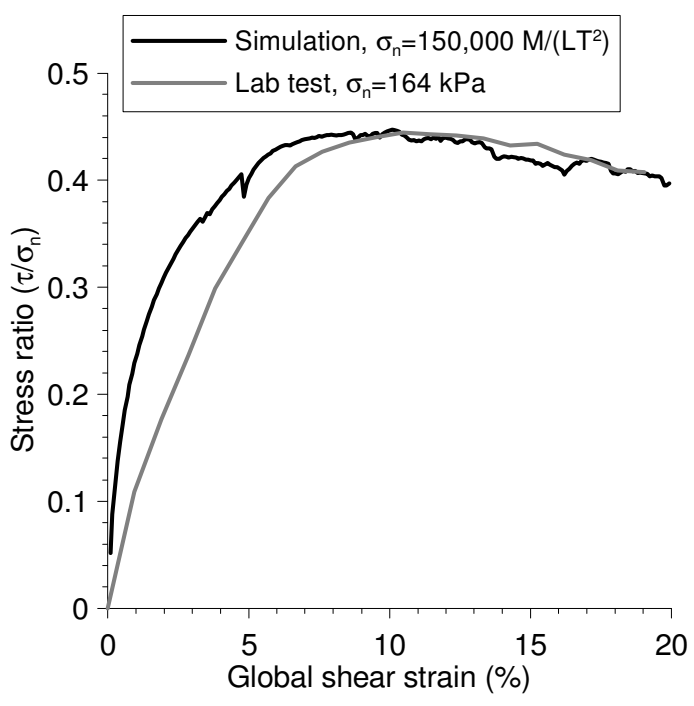

(a) Shear stress ratio versus global shear strain

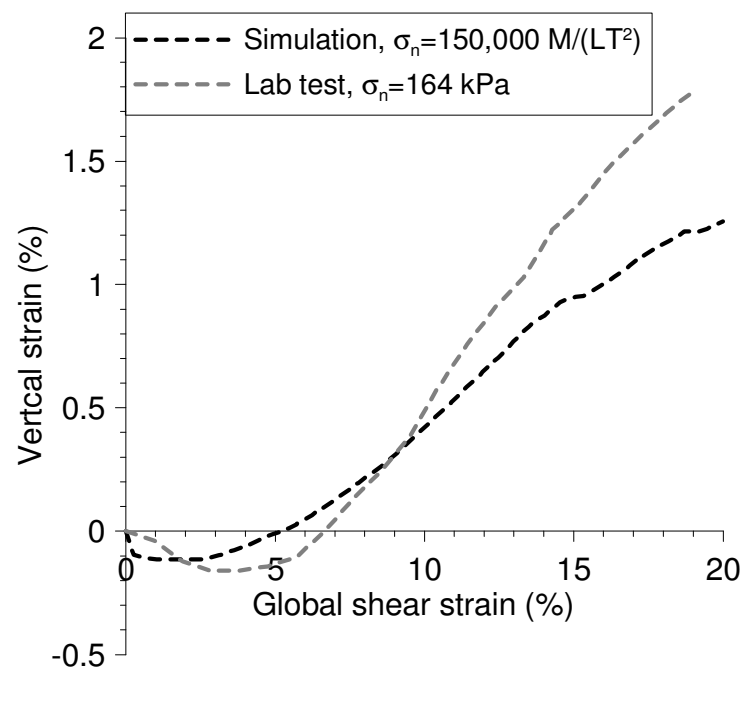

(b) Vertical strain versus global shear strain

Figure 10. Comparison of physical test results and DEM simulation for a representative direct shear test.

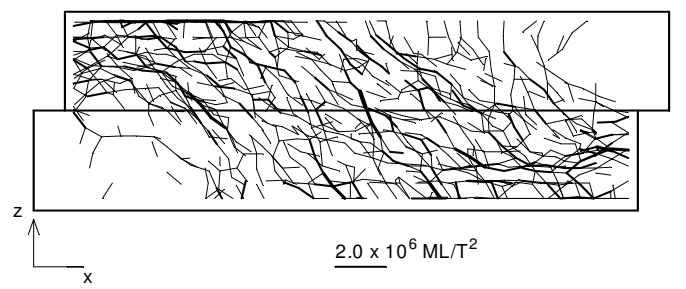

(a) Side view (looking along y axis)

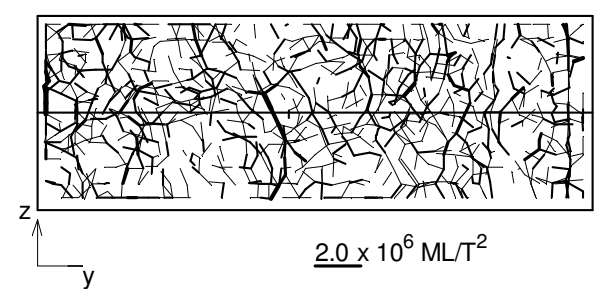

(b) Front view (looking along $\mathrm{x}$ axis)

Figure 11. Contact force network at $\gamma$ of $15.3 \%$ for representative DEM simulation of direct shear test

Two orthogonal views of the particle displacements are illustrated in Figure 12, considering the interval from $\gamma=$ $1.5 \%$ to $\gamma=15.3 \%$. For ease of visualization only displacements exceeding the average incremental displacement for the central fifth segment of the specimen are considered in Figure 12(a) and only the displacements exceeding the average incremental displacement plus one standard deviation for the whole specimen are plotted in Figure 12(b). Referring firstly to Figure 12(a), most of the displacement is concentrated in the upper half of the shear box, as would be expected. At the left hand side of the box there is downward motion of the particles, while at the right hand side of the box there is upward motion of the particles. Comparing Figure 12(a) and Figure 12(b), it is clear that the components of the particle displacement vectors in the y-direction are small but finite in comparison to the component of these vectors in the x-direction. Most of the displacement is concentrated in the upper half of the box. The movements of the particles are quite erratic in the shear "zone" across the centre of the box with the displacements in the y-direction of the particles in the shear zone being greater than in other regions. 


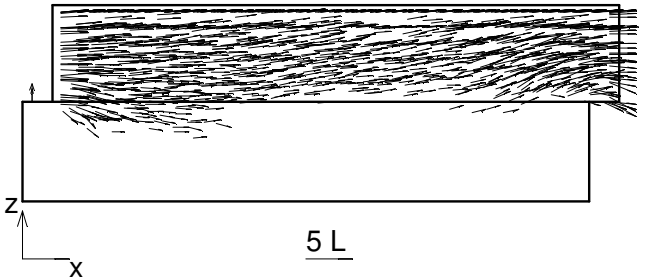

(a) Side view (looking along y axis)

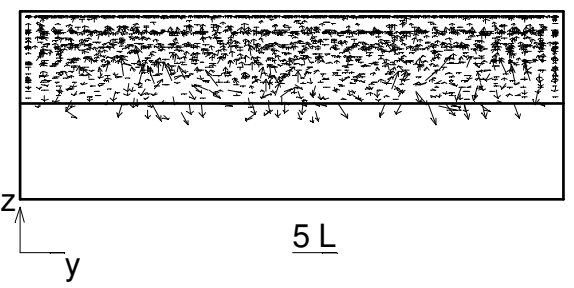

(b) Front view (looking along $\mathrm{x}$ axis)

Figure 12: Incremental displacement vectors for the interval from $\gamma=1.5 \%$ to $\gamma=15.3 \%$

\section{Conclusions}

Discrete element modeling is a valuable tool for geotechnical engineers who wish to analyze the micro-mechanisms underlying complex aspects of soil response. This paper has presented the results of a series of studies that have coupled physical tests with discrete element modeling to validate the ability of a relatively simple discrete element model to capture the real physical response. The principal findings of this study are:

1. The studies considering the response of disks and spheres with regular packing configurations have shown that once the particle characteristics (geometry and friction) are accurately measured and incorporated in the DEM model, the material response can be replicated accurately. This demonstrates that the simple rheological model, discussed above and illustrated in Figure 1, can be used in analysis of particulate materials.

2. Care should be taken when using dense, regular arrangements of uniform disks or uniform spheres in DEM code validation studies as the response of these systems is very sensitive to small changes in geometry.

3. Where random assemblies of particles are used in validation studies, a sufficiently large number of particles needs to be used to minimize sensitivity to minor geometrical variations.

4. The relationship between specimen strength and coordination number demonstrates that when a DEM analysis is carefully coupled with a series of physical tests, features of the macro-scale response can be explained from a micro-scale perspective.

5. The study of the direct shear test has demonstrated that DEM can capture the response of a random arrangement of particles, subject to a finite deformation, relatively accurately.

6. The micro-scale analysis of the direct shear test has shown that the earlier two-dimensional analyses of the direct shear test are qualitatively representative of the material response. However the contact forces and particle displacements orthogonal to the plane of shearing are finite, illustrating clearly the threedimensional nature of the material response.

Overall, there is much promise for us to examine in detail the laboratory test procedures that are routinely used in geotechnical design and analysis using DEM, both to better understand the observed material response and the limitations of the test procedures.

\section{Acknowledgements}

Funding for this research was provided by The O'Reilly Foundation and IRCSET (The Irish Research Council for Science Engineering and Technology). Some additional funding was provided by the David and Lucile Packard Foundation and the President's Research Fund of University College Dublin (UCD). Dr. Mike Reimer (UC Berkeley) and Mr. George Cosgrave (UCD) provided assistance when carrying out the laboratory tests.

\section{References}

Barla, M. and Barla, G. (2005) "Assessing design parameters for tunneling in a cemented granular soil by continuum and discontinuum modeling" in Prediction, Analysis and Design in Geomechanical Applications, proceedings of the $11^{\text {th }}$ International Conference of IACMAG, Barla, G. and Barla, M. (eds.) pp 475-484.

Cheng, YP, Nakata, Y, Bolton, MD. (2003) “Discrete element simulation of crushable soil” Geotechnique Vol. 53, No. 7, pp 633-641. 
Cheng, YP, Bolton, MD, Nakata, Y (2004) "Crushing and plastic deformation of soils simulated using DEM" Geotechnique Vol. 54, No. 2, pp 131-141.

Cui, L. and O'Sullivan, C. (2005) "Exploring the macro- and micro-scale response characteristics of an idealized granular material in the direct shear apparatus" Submitted to Geotechnique for publication, July 2005.

Cundall, P.A. and Strack, O.D.L. (1979) “A distinct element model for granular assemblies" Geotechnique, Vol. 29, No. 1, pp 47-65.

Dolezalova, M., Czene, P., and Havel, F. (2002) "Micromechanical modeling of stress path effects using PFC2D code" in Numerical Modeling in Micromechanics via Particle Methods, Proceedings of the $1^{\text {st }}$ International PFC Symposium, Konietzky, H. (ed), Balkema. pp 173-182

Dounias, G.T. and Potts, D.M. (1993) "Numerical analysis of drained direct and simple shear test" Journal of Geotechnical Engineering, ASCE Vol. 119, No. 12, pp 1870-1891.

García-Rojo, R., Herrmann, H.J. and S. McNamara, (Eds.) (2005) Powders and Grains 2005 A.A.Balkema, Rotterdam, 2005.

Horne, M.R. (1969) "The behaviour of an assembly of round, rigid, cohesionless particles III" Proceedings of the Royal Society, A310, pp 21-34.

Itasca Consulting Group (1993-1998). "PFC2D 2.00 Particle Flow Code in Two Dimensions". Itasca Consulting Group, Inc., Minneapolis, Minnesota.

Ke, T.-C., and Bray J.D. (1995). "Modeling of particulate media using discontinuous deformation analysis" Journal of Engineering, ASCE, Vol. 121 No. 11, pp 1234-1243.

Lanier, J. and Jean, M. (2000) "Experiments and numerical simulations with 2D disks assembly" Powder Technology Vol. 109, No. 1-3, pp 206-221.

Lin, X. and $\mathrm{Ng}$, T.-T. (1997) "A three-dimensional discrete element model using arrays of ellipsoids", Geotechnique, Vol. 47, No. 2, pp 319-329.

Ng, T.-T. (2004) "Shear strength of assemblies of ellipsoidal particles" Geotechnique Vol. 54, No. 10, pp 659-670.

Masson, S. and Martinez, J. (2001) "Micromechanical analysis of the shear behavior of a granular material" ASCE Journal of Engineering Mechanics, Vol. 127, No. 10, pp 1007-1016.

Oda, M., Takemura, T., and Takahashi, M. (2004) "Microstructure in shear band observed by microfocus X-ray computed tomography" Geotechnique Vol. 54, No. 8, pp 539-542.

O'Sullivan, C. (2002). The Application of Discrete Element Modelling to Finite Deformation Problems in Geomechanics. Ph.D. thesis, Dept. of Civ. Engrg., Univ. of California, Berkeley.

O'Sullivan, C., Bray, J.D. and M.F. Riemer, (2004) "An examination of the response of regularly packed specimens of spherical particles using physical tests and discrete element simulations." Journal of Engineering Mechanics, ASCE Vol. 130, No. 10, pp 1140-1150.

O'Sullivan, C. and J. D. Bray (2003) "Selecting a suitable time-step for discrete element simulations that use the central difference time integration approach." Engineering Computations Vol. 21, No. 2-4, pp. 278 - 303.

O’Sullivan, C., Bray, J. D. and Li, S. (2003) "A New Approach for Calculating Strain for Particulate Media" International Journal for Numerical and Analytical Methods in Geomechanics Vol. 27, No. 10, pp 859-877.

O'Sullivan, C. and J.D. Bray (2003) "A Modified Shear Spring Formulation for Discontinuous Deformation Analysis of Particulate Media" Journal of Engineering Mechanics, ASCE Vol. 129, No. 7, pp 830-834.

O'Sullivan, C., J.D. Bray, and M.F. Riemer, (2002) "The Influence of Particle Shape and Surface Friction Variability on Macroscopic Frictional Strength of Rod-Shaped Particulate Media" Journal of Engineering Mechanics, ASCE Vol. 128, No. 11. pp 1182-1192.

Rowe, P.W. (1962). "The stress-dilatancy relation for static equilibrium of an assembly of particles in contact." Proceedings of the Royal Society of London. Series A, Mathematical and Physical Sciences, Volume 269 No 1339, pp 500-527.

Skinner, A.(1969) "A note on the influence of interparticle friction on the shearing strength of a random assembly of spherical particles" Geotechnique Vol. 19, No.1, pp 150-157.

Thomas, P.A. (1997) Discontinuous deformation analysis of particulate media. Ph.D. thesis, Dept. of Civ. Engrg., Univ. of California, Berkeley.

Thomas, P.A. and Bray, J.D. (1999) "Capturing nonspherical shape of granular media with disk clusters" J. Geotecnical and Geoenvironmental Engineering, ASCE, Vol. 125, No. 3, pp 169-178.

Thornton, C (2000). "Numerical simulation of deviatoric shear deformation of dranular media." Geotechnique Vol 50, No. 1, pp 43-53. 
Thornton, C. (1979), "The conditions for failure of a face-centered cubic array of uniform rigid spheres", Geotechnique, Vol. 29, No. 4, pp 441-459.

Wang, L.B., Frost, J.D., and Lai, J.S., (2004), "Three-dimensional digital representation of granular material microstructure from X-ray tomography imaging", Journal of Computing in Civil Engineering ASCE, Vol. 18, No. 1, pp. 28-35.

White D.J., Take W.A. \& Bolton M.D. (2003) "Soil deformation measurement using particle image velocimetry (PIV) and photogrammetry". Geotechnique Vol. 53, No. 7, pp 619-631.

Zhang, L. and Thornton, C. (2002). "DEM simulations of the direct shear test". Proceedings of $15^{\text {th }}$ ASCE Engineering Mechanics Conference, June2-5, 2002. Columbia University, New York, NY. 